\title{
Influence of needle-insertion depth on epidural spread and clinical outcomes in caudal epidural injections: a randomized clinical trial
}

This article was published in the following Dove Press journal: Journal of Pain Research

\author{
Sang Jun Park ${ }^{1,2}$ \\ Kyung Bong Yoon ${ }^{1,2}$ \\ Dong Ah Shin ${ }^{3}$ \\ Kiwook Kim' \\ Tae Lim Kim' \\ Shin Hyung Kim ${ }^{1,2}$ \\ 'Department of Anesthesiology and \\ Pain Medicine, Yonsei University \\ College of Medicine, Seoul, Republic \\ of Korea; ${ }^{2}$ Anesthesia and Pain \\ Research Institute, Yonsei University \\ College of Medicine, Seoul, \\ Republic of Korea; ${ }^{3}$ Department \\ of Neurosurgery, Yonsei University \\ College of Medicine, Seoul, Republic \\ of Korea
}

Background: A caudal epidural steroid injection (CESI) is a commonly used method to improve symptoms of lumbosacral pain. We compared the achievement of successful epidurograms and patient-reported clinical outcomes following different needle-insertion depths during CESI.

Methods: For the conventional method group, the needle was advanced into the sacral canal. For the alternative method group, the needle was positioned immediately after penetration of the sacrococcygeal ligament. Epidural filling patterns and vascular uptake during fluoroscopy were determined to verify successful epidural injection. Procedural pain scores were investigated immediately after the procedure. Pain scores and patient global impression of symptom change were evaluated at 1-month follow-up.

Results: Assessments were completed by 127 patients (conventional method, $n=64$; alternative method, $n=63$ ). The incidence of intravascular injection was significantly lower in the alternative method group than in the conventional method group ( $3.2 \%$ vs $20.3 \%, P=0.005)$. Procedural pain during needle insertion was significantly lower in the alternative method group $(3.7 \pm 1.3$ vs $5.3 \pm 1.2, P<0.001)$. Epidural contrast filling patterns were similar in both groups. One-month follow-up pain scores and patient global impression of symptom change were comparable in both groups.

Conclusion: Compared with the conventional method, the alternative method for CESI could achieve similar epidural spread and symptom improvement. The alternative technique exhibited clinical benefits of a lower rate of intravascular injection and less procedural pain.

Keywords: caudal block, epidurogram, fluoroscopy, intravascular injection, pain, sacral canal

\section{Introduction}

Caudal epidural steroid injection (CESI) is a commonly used treatment option for patients with lumbosacral pain. ${ }^{1}$ Traditionally, CESI was performed by placing a needle through the sacral canal to deliver medications into the epidural space. This is a blind technique simply performed by palpating the sacral hiatus. However, inadvertent vascular injection is more common than with lumbar epidural injections, resulting in an increased risk of complications and ineffective injection. ${ }^{2,3}$ In addition, there is a potential risk of dural puncture, as the end of the dural sac may extend below the S3 level. ${ }^{4}$

With the advent of fluoroscopy and ultrasound in guiding needle placement, the success rates of caudal epidural block have improved dramatically. ${ }^{5,6}$ Doo et al eported an alternative approach, injecting medications immediately after penetration of the sacrococcygeal ligament, in which placing the needle into the sacral canal becomes unnecessary. ${ }^{7}$ This study demonstrated reduced rates of intravascular injection using the
Correspondence: Shin Hyung Kim Department of Anesthesiology and Pain Medicine, Yonsei University College of Medicine, 50 Yonsei-ro, Seodaemun-gu, Seoul I20-752, Republic of Korea

Tel +82 222287500

Fax +82 2364295 I

Email TESSAR@yuhs.ac 
real-time fluoroscopy and manual blood aspiration method. However, the detailed extent of epidural spread, such as cephalad spread and nerve root involvement, was not compared with the conventional approach. Furthermore, clinical benefits of the alternative CESI technique on procedural pain and patient-reported symptom improvement after the procedure were not investigated.

In this study, we used two different needle-insertion depths (into the sacral canal vs immediately after sacrococcygeal ligament penetration) for CESI, and compared epidurogram patterns and the incidence of intravascular injection using digital subtraction angiography (DSA). Needle-related pain during the procedure and Patient Global Impression of Change (PGIC) in symptoms at 1-month follow-up were investigated. Ultimately, we determined the clinical reliability of this alternative approach compared with the conventional approach for CESI.

\section{Materials and methods}

\section{Study population and randomization}

This randomized prospective clinical trial was approved by our institutional review board (4-2016-1030) and registered at ClinicalTrials.gov (NCT 03057197). Written informed consent was provided by each patient before study enrollment. The study was conducted at the outpatient department for pain management at Yonsei University College of Medicine,
Seoul, Republic of Korea, between March 2017 and March 2018. This manuscript adheres to the applicable CONSORT guidelines for randomized controlled studies.

The study included 130 patients (20-80 years of age) scheduled for CESI. Patients with general contraindications for fluoroscopy-guided injection, such as pregnancy, contrast material allergy, and coagulopathy, were excluded. Each patient was randomly assigned to either the conventional method group or the alternative method group. Randomization was performed using a computer-generated randomization sequence (http://www.randomizer.org) by an investigator who was not involved in the procedures. The different CESI methods were used for each patient based on the group assignment (Figure 1).

\section{Caudal epidural injections and outcome measures}

All procedures were performed using the same $\mathrm{C}$-arm fluoroscopy system (ARCADIS Varic 2013 model; Siemens Medical Solutions, Erlangen, Germany). A single practiced pain physician with 5 years of experience performed all procedures. The clinician performed CESIs after being given information on which depth (conventional or alternative) should be used. Two other researchers, not involved in performing the procedure, checked whether the contrast medium spread intravascularly, and observed the epidural

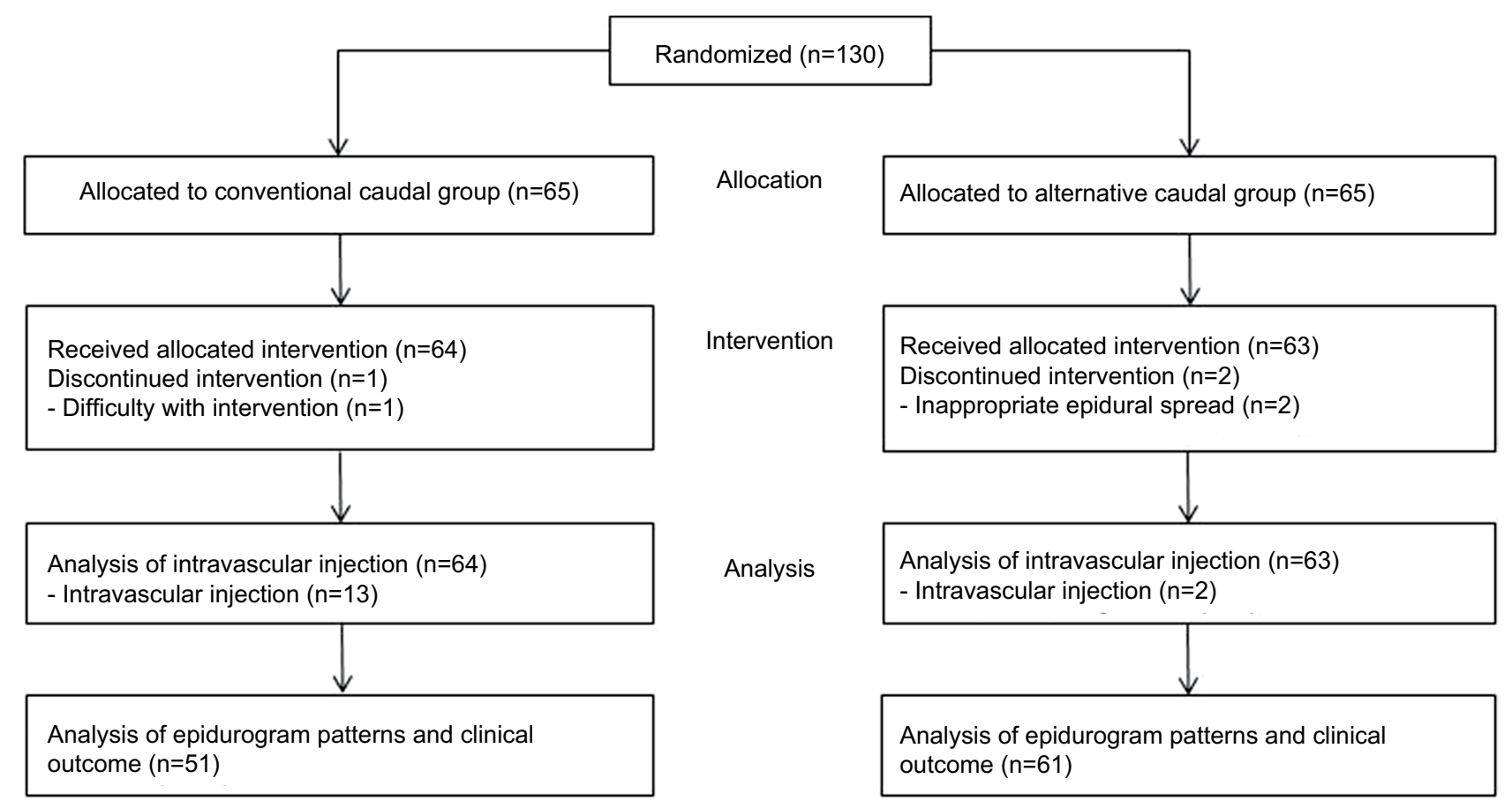

Figure I The CONSORT flow diagram. 
spread pattern during the process. The patients were placed in the prone position with a pillow beneath the lower abdomen and then covered with a sterile drape. The sacral hiatus was identified in the lateral fluoroscopy view as an abrupt dropoff at the caudal end of the S4 lamina. After infiltration of the skin at the planned needle entry point with $1 \%$ lidocaine, a spinal needle ( $22 \mathrm{G}, 8 \mathrm{~cm}$ Quincke) was inserted into the epidural space through the sacral hiatus using intermittent fluoroscopic guidance. In the conventional method group, the needle was inserted into the sacral canal and advanced to the mid-S3 level. In the alternative method group, the needle tip was inserted into the epidural space until a "pop" was felt as the sacrococcygeal ligament was penetrated; the sacral canal was unaffected (Figure 2).

After verification of the final needle position using lateral and anteroposterior (AP) views, the needle was attached to an extension tube, which was connected to a $5 \mathrm{~mL}$ syringe at the opposite end. The plunger of the syringe was withdrawn to check for blood. If this aspiration test was negative, $1 \mathrm{~mL}$ of contrast medium was slowly injected at $0.1 \mathrm{~mL} / \mathrm{second}$; DSA was used to assess intravascular and sacral epidural space injection. Intravascular injection was characterized by the appearance and immediate disappearance of contrast medium in a snake-like pattern. Each distribution pattern was assigned to one of three categories: epidural only, epidural and intravascular, or intravascular only. If intravascular spread of contrast medium was observed, the needle was repositioned and the lack of vascular uptake was confirmed. When no vascular flow was observed, $15 \mathrm{~mL}$ of injectate $(0.2 \%$ lidocaine with $5 \mathrm{mg}$ dexamethasone disodium phosphate and $5 \mathrm{~mL}$ contrast medium) was injected, then the pattern of contrast distribution was observed under fluoroscopy.
Dispersion of contrast medium into the epidural space and filling of nerve roots was observed in AP and lateral views.

We collected patient demographic and clinical data, including age, gender, weight, height, body mass index, pain score, duration of pain, and main diagnoses, and previous spinal surgery history. Procedural pain was investigated separately from the existing (pre-procedure) pain, using a ten-point numeric rating scale from $0=$ no pain to $10=$ worst imaginable pain. We defined procedural pain as pain from the start of needle insertion until it reached its final position. Patients rated the procedural pain immediately after the CESI was completed. At 1-month follow-up, we evaluated patient-reported pain scores and overall symptom improvement (PGIC: $1=$ very much improved, $2=$ much improved, $3=$ minimally improved, $4=$ no change, $5=$ minimally worse, $6=$ much worse, and $7=$ very much worse). An independent observer who was not involved in the procedure confirmed and recorded all assessments in this study.

\section{Statistical analysis}

The primary endpoint of this study was the success rate of epidural spread without intravascular uptake. The results of a previous study revealed a $41.7 \%$ incidence of intravascular injection for CESI, when verified by DSA. ${ }^{8}$ We considered a $55 \%$ decrease in the rate of intravascular injection with the alternative method of CESI to be clinically relevant. Power analysis results indicated that a sample size of 65 patients was required for each group $(\alpha$-error $=0.05$, power $=80 \%$, dropout rate $=5 \%$ ). All results are expressed as mean $\pm \mathrm{SD}$ or number of patients. The Shapiro-Wilk test was used to determine whether data were normally distributed. The Student's $t$-test, chi-squared test, or Mann-Whitney $U$ test was used where
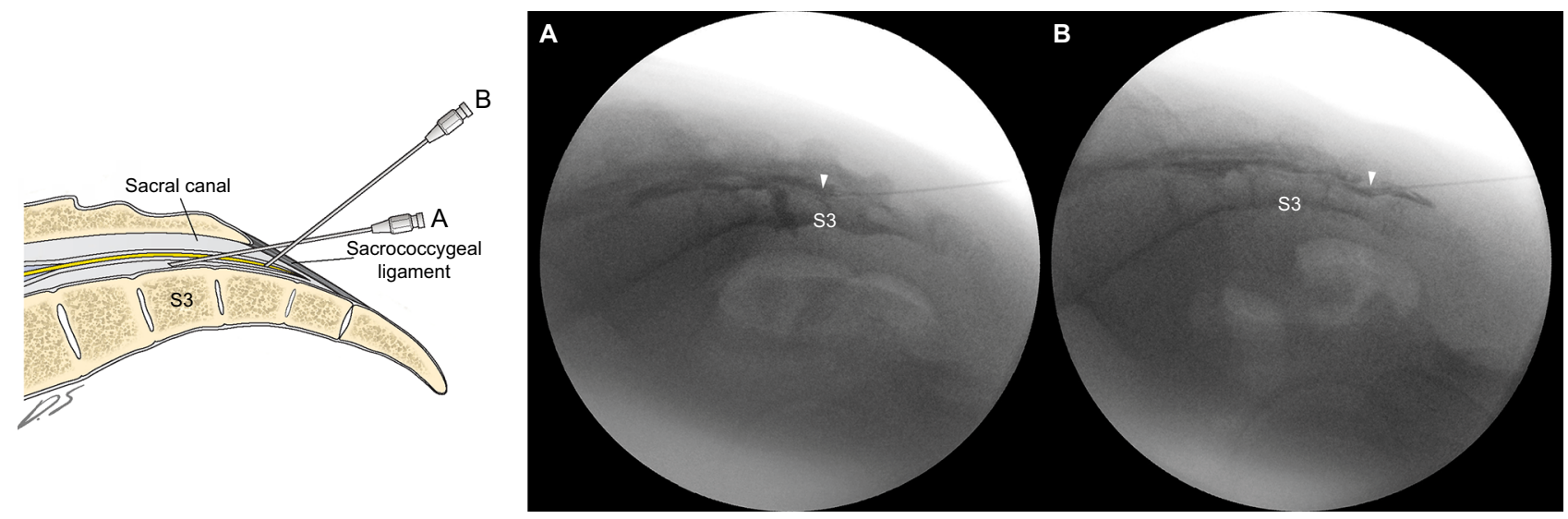

Figure 2 Schematic diagram and fluoroscopic images demonstrating needle placement of $(\mathbf{A})$ the conventional method and (B) the alternative method for caudal epidural injection.

Note: S3, third vertebral body of sacral spine; arrowheads indicate the tip of the needle. 
appropriate for between-group comparisons of demographic and clinical data. SPSS version 23 (IBM Corp., Armonk, NY, USA) was used for all analyses. Results with a $P$-value $<0.05$ were considered to be statistically significant.

\section{Ethics}

This trial was conducted in accordance with the Declaration of Helsinki.

\section{Results}

We enrolled and randomized 130 patients. Three patients were excluded from the study population for the final analysis. One patient in the conventional method group was converted to the alternative method group because of difficulty approaching the sacral canal. Two patients from the alternative method group were converted to the conventional method group because the contrast medium was mostly observed at the coccygeal level, and thus had failed to ascend to the cephalad epidural space. Therefore, data from 64 patients in the conventional method group and 63 patients in the alternative method group were analyzed. In 13 patients in the conventional method group and two in the alternative method group, intravascular uptake was observed, and needle repositioning or a second attempt at the epidural injection was subsequently necessary during the procedure. These patients were excluded in the analysis of epidurogram patterns and the clinical outcomes at 1-month follow-up (Figure 1).

Patient characteristics and baseline clinical data, including pre-procedure pain scores, are presented in Table 1 . The rate of successful epidural spread was significantly higher in the alternative method group than in the conventional method group ( $96.8 \%$ vs $79.7 \%, P=0.005)$. The incidence of intravascular injection was significantly lower in the alternative method group (3.2\% vs $20.3 \%, P=0.005)$ (Table 2 ). Procedural pain during needle insertion was significantly lower in the alternative method group $(3.7 \pm 1.3$ vs $5.3 \pm 1.2$, $P<0.001)$. The epidural and nerve root filling patterns are presented in Table 3. Most patients (93\%) exhibited ventral filling extending up to the L5-S1 level. Ventral filling and nerve root filling were not significantly different between the two groups. While pain scores in both groups were reduced at 1-month follow-up, there was no statistically significant difference in post-procedure pain relief between the two groups ( $1.7 \pm 1.3$ vs $1.7 \pm 1.6, P=0.913$ ) (Figure 3 ). Similarly, the median value of PGIC was 3 (minimally improved) in

Table 2 Incidence of intravascular injections

\begin{tabular}{|l|l|l|l|}
\hline & $\begin{array}{l}\text { Conventional } \\
(\mathbf{n}=64)\end{array}$ & $\begin{array}{l}\text { Alternative } \\
(\mathbf{n}=63)\end{array}$ & $\boldsymbol{P}$-value \\
\hline Epidural only & $51 / 64(79.7)$ & $61 / 63(96.8)$ & 0.004 \\
Intravascular & $13 / 64(20.3)$ & $2 / 63(3.2)$ & 0.005 \\
$\begin{array}{l}\text { Epidural and } \\
\text { intravascular }\end{array}$ & $12 / 13(92.3)$ & $2 / 2(100)$ & \\
Intravascular only & $1 / 13(7.7)$ & $0(0)$ & \\
\hline
\end{tabular}

Note: Values are expressed as number (\%) of patients.

Table 3 Analysis of epidurogram patterns

\begin{tabular}{|c|c|c|c|}
\hline Type of spread & $\begin{array}{l}\text { Conventional } \\
(n=51)\end{array}$ & $\begin{array}{l}\text { Alternative } \\
(n=61)\end{array}$ & $P$-value \\
\hline \multicolumn{4}{|l|}{ Ventral spread } \\
\hline L5-SI level & 48/5I (94.I) & $54 / 61(88.5)$ & 0.722 \\
\hline L4-5 level & $|5 / 5|(29.4)$ & $|4 / 6|(22.9)$ & 0.523 \\
\hline \multicolumn{4}{|l|}{ Nerve root spread } \\
\hline SI root & $27 / 5 \mid(52.9)$ & $32 / 61$ (52.4) & 1.000 \\
\hline L5 root & 4/5I (7.8) & $3 / 61$ (4.9) & 0.702 \\
\hline
\end{tabular}

Note: Data are presented as number of cases with spreading/total number of cases in the group (\% of cases with spreading).

Table I Patient characteristics and baseline clinical data

\begin{tabular}{|l|l|l|l|}
\hline & Conventional $(\mathbf{n}=\mathbf{6 5})$ & Alternative $(\mathbf{n}=\mathbf{6 5})$ & $\boldsymbol{P}$-value \\
\hline Gender (female/male) & $40 / 25$ & $35 / 30$ & 0.375 \\
\hline Age (years) & $65.6 \pm 10.5$ & $65.1 \pm 11.4$ & 0.810 \\
\hline Body mass index $\left(\mathrm{kg} / \mathrm{m}^{2}\right)$ & $24.3 \pm 2.7$ & $24.8 \pm 3.5$ & 0.363 \\
\hline Baseline pain scores (NRS) & $6.8 \pm 1.6$ & $6.7 \pm 1.8$ & 0.643 \\
\hline Pain duration (months) & $5.5 \pm 4.2$ & $4.6 \pm 3.4$ & 0.207 \\
\hline Lumbar spine surgery history & $45(69.2)$ & $46(70.7)$ & 0.848 \\
\hline Diagnosis & $29(44.6)$ & $32(49.2)$ & 0.725 \\
\hline Spinal stenosis & $15(23.0)$ & $9(13.8)$ & 0.258 \\
\hline Herniated lumbar disc & $16(24.6)$ & $11(16.9)$ & 0.387 \\
\hline Post-spinal surgery syndrome & $5(7.6)$ & $13(20.0)$ & 0.073 \\
\hline Radiculopathy of other origin & & \\
\hline
\end{tabular}

Notes: Values are expressed as mean \pm SD, number, or number (\%) of patients. NRS; 0-10.

Abbreviation: NRS, numeric rating scale. 


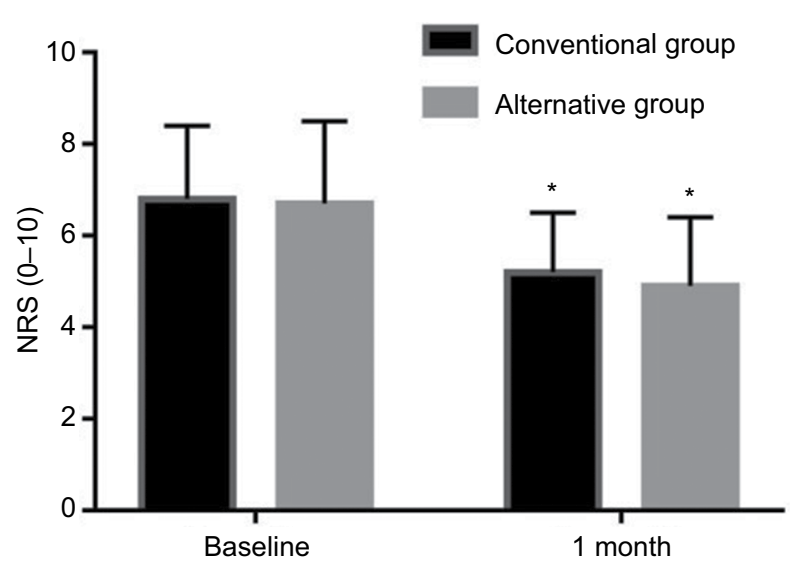

Figure 3 Changes in pain scores during the study period.

Notes: Values are expressed as mean \pm SD. $* P<0.05$ vs baseline in each group. There was no significant difference in pain scores between the two groups at I month after injection $(P=0.333)$.

Abbreviation: NRS, numeric rating scale.

Table 4 PGIC at I-month follow-up

\begin{tabular}{|l|l|l|}
\hline PGIC rating & $\begin{array}{l}\text { Conventional } \\
(\mathbf{n = 5} \text { I) }\end{array}$ & $\begin{array}{l}\text { Alternative } \\
(\mathbf{n = 6} \text { I) }\end{array}$ \\
\hline I. Very much improved & $0(0)$ & $0(0)$ \\
2. Much improved & $13(25.5)$ & $17(27.9)$ \\
3. Minimally improved & $32(62.7)$ & $34(55.7)$ \\
4. No change & $6(11.8)$ & $10(16.4)$ \\
5. Minimally worse & $0(0)$ & $0(0)$ \\
6. Much worse & $0(0)$ & $0(0)$ \\
7. Very much worse & $0(0)$ & $0(0)$ \\
\hline
\end{tabular}

Notes: Values are expressed as number of patients (\%). The Mann-Whitney $U$ test showed no significant difference in PGIC ratings between the two groups $(P=0.889)$. Abbreviation: PGIC, Patient Global Impression of Change.

both groups $(P=0.889)$ (Table 4$)$. There were two cases of facial edema, which was possibly a corticosteroid side effect. No severe episodes, such as dural puncture, were reported.

\section{Discussion}

In the present study, compared with the conventional method, the alternative method for CESI could achieve similar epidural spread and symptom improvement. The alternative technique exhibited clinical benefits of a lower rate of intravascular injection and less procedural pain.

In caudal epidural injections, intravascular injection increases the likelihood of complications and reduces the effectiveness of the procedure. ${ }^{2,3}$ Our results showed that during CESI, the incidence of intravascular injection was significantly lower with the alternative method than with the conventional method. There are two possible explanations for this difference. In the alternative technique, as the sacral canal is preserved from needling, bony contact with the needle is less likely. When the needle touches bone, it may penetrate or injure vessels near the bone surface. Shin et al reported that when the needle contacts bone between the posterior and anterior sacral foramina during S1 transforaminal epidural steroid injections, the intravascular injection rate increases, with an OR of 2.624. ${ }^{9}$ The second explanation involves the kyphotic nature of the sacrum and the needle-insertion angle. In the conventional technique, the needle is inserted at a shallow angle to the sacral canal where, in many cases, it comes into contact with the anterior wall of the sacral spine. The sacral venous plexus is located along the anterior wall of the sacral canal and usually terminates at S4 but may extend inferiorly, particularly in older patients..$^{10,11}$

There has been little research regarding procedural pain during caudal epidural injections. Previous studies reported post-injection pain as one of the adverse effects of caudal blocks. Ogoke reported that pain may persist at the sacral hiatus site of entry, but it usually resolves within 2-6 months and is associated with ecchymosis at the injection site. ${ }^{12}$ Another study reported injection-site soreness in $18 \%$ of patients after caudal epidural injection. ${ }^{3}$ In our study, compared with the conventional method, the alternative method was associated with lower procedural pain. This finding may be attributed to the fact that the pain-sensitive structures, such as sacral nerves, fat tissue, and bone, are secure from needling in the alternative approach.

In the present study, contrast agent spread to the coccygeal level in two out of 65 patients in the alternative method group, instead of ascending to the lumbosacral level. These patients excessively complained of pain during the procedure and were subsequently injected using the conventional method. The injectate failed to travel in the cephalad direction and stagnated at the coccygeal level outside the sacral canal, possibly because of anatomic variation, such as a very small sacral canal diameter. ${ }^{13}$ Conversely, one patient had a narrow sacral canal that was difficult to access by the conventional technique. When the alternative method was used instead, the injection was successful. Certain anatomic features and variations in the sacral hiatus may lead to difficult needle insertion into the caudal epidural space. A previous study reported that an AP diameter $<3.7 \mathrm{~mm}$ at the sacral hiatus apex was associated with difficulty inserting a needle into the caudal epidural space using the blind technique. ${ }^{14}$ Nikooseresht et al reported that the average AP diameter of the sacral hiatus apex in patients 
with failed caudal epidural needle insertion was $1.61 \pm 0.1$ $\mathrm{mm}$, which was significantly less than the diameter in patients with successful insertion $(4.7 \pm 1.7 \mathrm{~mm}) .{ }^{15}$ On the other hand, the extent of epidural spread confirmed by fluoroscopy during CESI may be associated with the clinical outcome after the procedure. ${ }^{16,17}$ There was no difference in ventral spread or nerve root filling between the two groups in the current study. Moreover, pain relief was mostly achieved at 1-month follow-up in both groups. Collectively, this study demonstrated that the alternative method may be a useful option when the conventional approach is difficult in clinical practice.

\section{Limitations}

There are some limitations in this study. First, the operator was not blinded to the injection method, although an independent observer who was not involved in the procedure recorded and confirmed the results. Second, this study used a real-world clinical practice model in which there were many potential confounders, such as medication type, which could have affected clinical outcomes. Third, we did not assess psychological factors which may have affected procedural pain and postprocedure clinical outcomes. Another limitation of this study is the short follow-up time of 1 month after the procedure.

\section{Conclusion}

This study confirmed that successful epidural spread can be achieved during CESI if the needle passes through the sacrococcygeal ligament but does not advance into the sacral canal. Moreover, this alternative technique was associated with a lower incidence of intravascular uptake and less procedural pain. Therefore, this study supports the use of ultrasound-guided CESI with the alternative approach. Although needle placement within the sacral canal may anatomically guarantee drug delivery into the epidural space, the alternative approach would be beneficial for selected patients in whom technical difficulty or excessive sensitivity to procedural pain is expected during CESI.

\section{Data sharing}

The authors are willing to share the data in this article. All of the individual participant data collected during the trial will be available, after deidentification. The data will be available for anyone who wishes to access the data for any purpose. Data will be available indefinitely at http://datadryad.org/. The data will be accessible immediately following publication, with no end date.

\section{Acknowledgment}

The authors would like to thank Dong-Su Jang, MFA (medical illustrator) for his help with the illustrations.

\section{Disclosure}

The authors report no conflicts of interest in this work.

\section{References}

1. Conn A, Buenaventura RM, Datta S, Abdi S, Diwan S. Systematic review of caudal epidural injections in the management of chronic low back pain. Pain Physician. 2009;12(1):109-135.

2. Sullivan WJ, Willick SE, Chira-Adisai W, et al. Incidence of intravascular uptake in lumbar spinal injection procedures. Spine. 2000;25(4): 481-486.

3. Manchikanti L, Cash KA, Pampati V, Mcmanus CD, Damron KS. Evaluation of fluoroscopically guided caudal epidural injections. Pain Physician. 2004;7(1):81-92.

4. Senoglu N, Senoglu M, Ozkan F, Kesilmez C, Kizıldag B, Celik M. The level of termination of the dural sac by MRI and its clinical relevance in caudal epidural block in adults. Surg Radiol Anat. 2013;35(7): 579-584.

5. Renfrew DL, Moore TE, Kathol MH, El-Khoury GY, Lemke JH, Walker CW. Correct placement of epidural steroid injections: fluoroscopic guidance and contrast administration. AJNR Am J Neuroradiol. 1991;12(5):1003-1007.

6. Chen CP, Wong AM, Hsu CC, et al. Ultrasound as a screening tool for proceeding with caudal epidural injections. Arch Phys Med Rehabil. 2010;91(3):358-363.

7. Doo AR, Kim JW, Lee JH, Han YJ, Son JS. A comparison of two techniques for ultrasound-guided caudal injection: the influence of the depth of the inserted needle on caudal block. Korean J Pain. 2015;28(2):122-128.

8. Fukazawa K, Matsuki Y, Ueno H, Hosokawa T, Hirose M. Risk factors related to accidental intravascular injection during caudal anesthesia. J Anesth. 2014;28(6):940-943.

9. Shin J, Kim YC, Lee SC, Kim JH. A comparison of Quincke and Whitacre needles with respect to risk of intravascular uptake in S1 transforaminal epidural steroid injections: a randomized trial of 1376 cases. Anesth Analg. 2013;117(5):1241-1247.

10. Martin L. Sacral epidural (caudal) block. In: Wildsmith J, Armitage E, editors. Principles and Practice of Regional Aanaesthesia. Edinburgh: Churchill Livingstone; 1987:102-103.

11. Bogduk N, Cherry D. Epidural corticosteroid agents for sciatica. Med J Aust. 1985;143(9):402-406.

12. Ogoke BA. Caudal epidural steroid injections. Pain Physician. 2000;3(3):305-312.

13. Kim DH, Park JH, Lee SC. Ultrasonographic evaluation of anatomic variations in the sacral hiatus: implications for caudal epidural injections. Spine. 2016;41(13):E759-E763.

14. Kim YH, Park HJ, Cho S, Moon DE. Assessment of factors affecting the difficulty of caudal epidural injections in adults using ultrasound. Pain Res Manag. 2014;19(5):275-279.

15. Nikooseresht M, Hashemi M, Mohajerani SA, Shahandeh F, Agah M. Ultrasound as a screening tool for performing caudal epidural injections. Iran J Radiol. 2014;11(2):e13262.

16. White AH, Derby R, Wynne G. Epidural injections for the diagnosis and treatment of low-back pain. Spine. 1980;5(1):78-86.

17. Manchikanti L, Staats PS, Singh V, et al. Evidence-based practice guidelines for interventional techniques in the management of chronic spinal pain. Pain Physician. 2003;6(1):3-81. 
The Journal of Pain Research is an international, peer reviewed, open access, online journal that welcomes laboratory and clinical findings in the fields of pain research and the prevention and management of pain. Original research, reviews, symposium reports, hypothesis formation and commentaries are all considered for publication
The manuscript management system is completely online and includes a very quick and fair peer-review system, which is all easy to use. Visit http://www.dovepress.com/testimonials.php to read real quotes from published authors. 